\title{
"POR HEPILA FAMOSA": \\ POSIBLE ALUSIÓN A JERÓNIMO DE URREA EN EL QUIJOTE DE 1605*
}

\author{
DAVID MAÑERo LozANo \\ Universidad Autónoma de Madrid
}

A comienzos de 1605 , dado el extraordinario éxito comercial de la primera edición del Quijote, agotada en apenas unas semanas, las prensas madrileñas de Juan de la Cuesta se apresuraron a imprimir una segunda edición del texto cervantino. Como es sabido, se introducen en ésta dos largos pasajes en los capítulos XXIII y Xxx, además de otras variantes que aún siguen suscitando no pocas discusiones críticas ${ }^{1}$.

No obstante, entre las enmiendas que gozaron de una mayor aceptación en las siguientes ediciones de la obra, destaca la lectura de un importante párrafo del primer capítulo, en el que la lección «por Hepila famosa» de la princeps (fol. $3 \mathrm{v}$, lín. 16) fue sustituida por otra radicalmente distinta, que resaltamos en cursiva:

Pero, acordándose que el valeroso Amadís no sólo se había contentado con llamarse Amadís a secas, sino que añadió el nombre de su reino y patria, por hacerla famosa, y se llamó Amadís de Gaula, así quiso, como buen caballero, añadir al suyo el nombre de la suya $[. . .]^{2}$.

\footnotetext{
* Agradecemos la información facilitada por Elena Varela, quien nos dio a conocer el importante testimonio del ms. 3660 de la BNM.

${ }^{1}$ Un reciente análisis sobre los problemas de edición de los textos cervantinos puede consultarse en Florencio Sevilla Arroyo, «La edición de las obras de Miguel de Cervantes, I», en Cervantes, Madrid, Centro de Estudios Cervantinos, 1995, págs. 75-101; con una segunda parte en págs. 103-35.

${ }^{2}$ Cfr. Martín de Riquer, ed. Miguel de Cervantes, El Ingenioso Hidalgo Don Quijote de la Mancha, Barcelona, Planeta, 1990,10. ${ }^{\mathrm{a}}$ ed., pág. 39; donde, según la práctica generalizada en la mayoría de las ediciones (vid. nota subsiguiente), se acepta la enmienda que parte de la segunda edición de 1605 . No falta, con todo, algún caso aislado en el que se edita a partir de la princeps, como en la ed. revisada y ampliada de Florencio Sevilla Arroyo y Antonio Rey Hazas (Miguel de Cervantes, El Ingenioso Hidalgo Don Quijote de la Mancha, Alcalá de Henares, Centro de Estudios Cervantinos, 1994, pág. 35).
}

RFE, LXXX, 2000, $1 .^{\circ}-2 .^{\circ}$, págs. $215-221$ 
Se solventa, con esto, la comprensión de un pasaje que, de modo sorprendente, no ha recibido hasta el momento la necesaria atención crítica ${ }^{3}$. Todo lo más, presuponiéndose, por su relativa dificultad de interpretación, la invalidez de la primera lectura, se encaminaron los esfuerzos de la crítica a reconstruir los motivos que llevarían al cajista a componer, a partir de «por hacerla famosa», la lectura supuestamente errónea de la princeps, que se vería restituida a su intención original ya en la segunda edición. El proceso ha sido expuesto con minuciosa atención por el profesor Francisco Rico, quien acumula una serie de argumentos ecdóticos a los que adjunta, como apoyo documental, un elocuente autógrafo cervantino.

Se nos excusará, entonces, si reproducimos al completo el largo párrafo dedicado al problema por el citado especialista:

Cervantes hubo de escribir que el espejo y flor de toda caballería "no se contentó con llamarse 'Amadís' a secas, sino que añadió el nombre de su reino y patria, por hácerla famosa, y se llamó "Amadís de Gaula". En la caligrafia del novelista, sin embargo, la zeta (y hazerla se escribía entonces con zeta) se prolonga mucho hacia abajo y, por ende, se presta a ser malinterpretada como una pe o una $y$ griega (échese un vistazo a la segunda línea del autógrafo anejo ${ }^{4}$ ), de suerte que, por ejemplo, un hizo del autor se entendió una vez como leyó. Una confusión de ese tipo se produjo sin duda al sacar a limpio (o a molde) el manuscrito cervantino: la zeta de hazerla se tomó por pe, y como en una voz tan frecuente el nexo con las letras contiguas estaría trazado tan veloz como imprecisamente, el resultado fue que la príncipe estampó "por Hepila famosa" (!)

En efecto, la explicación nos hace ver con absoluta nitidez la posibilidad de que la $z$ de ese hazerla que, hipotéticamente, encontraría el cajista a la hora de transcribir el desconocido manuscrito de Cervantes, fuese confundida con la grafía $p$ o, incluso, con la $y$, pero este despiste de lectura daría lugar, en principio, no a lo que leemos en la primera edición, Hepila, sino a un haperla o hayerla que aún dista mucho de la lectura de la princeps.

\footnotetext{
${ }^{3}$ Sin ningún tipo de anotación al texto, se edita «por hacerla famosa» en las eds. de Luis Andrés Murillo (Madrid, Castalia, 1986, 3 vols.); Luis Astrana Marín (Valencia, Ortells, 1993); Juan Bautista Avalle Arce (Madrid, Alambra, 1988, 2 vols.); Ángel Basanta (Barcelona, Plaza \& Janés, 1985, 2 vols.); Joaquín Casalduero (Madrid, Alianza, 1993, 2 vols.); Vicente Gaos (Madrid, Gredos, 1987, 3 vols.); Justo García Soriano y Justo García Morales (Madrid, M. Aguilar, 1947); Juan Ignacio Ferreras (Madrid, Akal, 1991, 2 vols.); John Jay Allen (Madrid, Cátedra, 1991, 2 vols.); Francisco Rodríguez Marín (Madrid, Atlas, 1947-9, 10 vols.); etc.

${ }^{4}$ El autógrafo en cuestión es un memorial de Cervantes a Felipe II, fechado en 1594, en el que figura el término hazer con un trazado de la $z$ que el investigador juzga afín al de $p$ o $y_{5}$

${ }^{5}$ Cfr. Francisco Rico, «"Por Hepila famosa” o cómo no editar el Quijote», Babelia, 14-91996, págs. 16-7; en particular, pág. 16 la cita.
} 
Es, entonces, de rigor, recordar el razonamiento de Alberto Blecua, para quien:

en buena ley textual, es imposible que un copista ni un componedor escriba "Hepila" donde pone "hazerla". O bien desconocemos qué significa "Hepila" o bien se trata de un error en esta palabra [...]. En todo caso, "hazerla" es una conjetura facilior de un componedor ${ }^{6}$.

En efecto, sería necesario, en caso de rechazar la observación de Blecua, justificar no sólo esa confusión de hazerla por haperla, sino también presuponer otras como Haperla, heperla, hapirla y hapila, errores todos que, de modo simultáneo, tendrían que operarse en la mala lectura del copista para sostener que éste, como previo paso a componer Hepila, habría encontrado hazerla en el autógrafo de Cervantes.

Pero no es nuestra intención tratar aquí de desmentir la prioridad de $\mathrm{He}$ pila desde el punto de vista textual. Lejos de esto, nos esforzaremos en avanzar por el camino opuesto, es decir, en intentar aportar algunos datos con los que poder afrontar un acercamiento semántico a la lectura de la princeps.

Atendamos, en primer término, a una de las obras dedicadas por Andrés de Ustarroz a la poesía aragonesa, en la que dedica una interesante silva a Jerónimo Jiménez de Urrea, natural de Épila. En transcripción paleográfica:

ÉPILA

Don Gerónimo claro el apellido

de Ximénez de Urrea, esclarecido

por el orbe derrama,

y coronado de laurel, y grama

5 sube a la cumbre excelsa del Parnaso

competidor del dulce Garcilaso.

Este, que en nuestro idioma numeroso

el Orlando traduxo furioso

y el Carlos victorioso

10 escribió del heroico Carlos quinto

aunque en Mapa sucinto

las vitorias, y hazañas,

que consiguió en provincias tan estrañas.

Clarisel de las flores

15 contiene suavísimos amores, y la Epilia famosa

\footnotetext{
${ }^{6}$ Cfr. Alberto Blecua, en F. Brioschi y C. Di Girolamo, Introducción al estudio de la literatura, Barcelona, Ariel, 1988, pág. 40, donde, sin apartarse radicalmente de la princeps, se sugiere que «podría tratarse de hepilasi o hepilesi del griego hepilepsis, esto es, cognominatio en latín y sobrenombre en la época de Cervantes».
} 
de Epila su patria gloriosa

las grandezas contiene.

Los discretos pastores,

20 que en su Arcadia Sanazaro cantaba

al español idioma trasladaba,

que su ingenio perene

fue gloria numerosa de Pirene ${ }^{7}$.

En efecto, se constata en los vv. 16-18, bien que $\sin H^{8}$, el recuerdo aún vivo de la Epilia famosa de Jerónimo Jiménez de Urrea, obra que, quizá por permanecer inédita, ha sido hasta el momento silenciada por buena parte del hispanismo, sin que falte, siempre al margen de las ediciones y estudios de Cervantes, alguna que otra alusión como la de Latassa, quien, al recensar el conjunto de las obras del poeta aragonés, recoge, invirtiendo el título de la obra, idénticos datos a los de Ustarroz, de donde parece extraer su información:

La famosa Epila. Obra que trabajó en obsequio de su patria para celebrar sus grandezas, imitando la Arcadia de Sanazaro?.

A la vista de estos datos, consideramos oportuno apuntar que, más allá de los traídos y llevados despistes de impresores o cajistas, cuando no de los atribuidos al propio Cervantes, el mencionado párrafo del Quijote parece aludir a la obra de Urrea, máxime si reparamos en que, en ésta, al igual que Amadís «añadió el nombre de su reino y patria», se destaca por partida doble -observación coincidente en Ustarroz y Latassa - el deseo de engrandecer la «patria», precisamente el término anterior a «por Hepila famosa».

Por otra parte, se sostiene con esta hipótesis la mención a una obra muy poco conocida, que, como en otros $\operatorname{casos}^{10}$, quizá encerró un contenido só-

\footnotetext{
${ }^{7}$ Cfr. Juan Francisco Andrés de Ustarroz, Aganipe de los cisnes aragoneses celebrados en el Clarín de la fama por el Dr. Juan Francisco Andrés. 1781, Zaragoza, Tip. de Comas hermanos, 1890, pág. 123. Según hemos comprobado, esta edición recoge, con variantes gráficas de escasa relevancia y omisión del verbo «cantaba» de v. 20, el poema autógrafo de Ustarroz contenido en el f. 209 del ms. 3660 de la BNM.

${ }^{8} \mathrm{Sin}$ ir más lejos, en el f. $273 \mathrm{v}$ del ms. 9457 de la BNM, autógrafo de Ustarroz del que después nos ocuparemos, aparece escrito con grafia $H$ el término 'Ebro', lo que nos ofrece una entre tantas muestras del caprichoso empleo de esta grafía en la época. En cuanto al uso en Cervantes del término Hepila, en el que se observa una monoptongación respecto de la forma Epilia con la que habitualmente citan otros autores la obra de Urrea, creemos que la existencia del topónimo Epila es ya motivo más que suficiente para pensar en una lectio facilior de Epilia por Hepila.

${ }^{9}$ Cfr. Félix de Latassa, Biblioteca nueva de escritores aragoneses, Pamplona, Joaquín de Domingo, 1798, I, pág. 268.

${ }^{10}$ Recuérdense, sin ir más lejos, los ataques lanzados a Lope en los preliminares de la primera parte, a los que habrá después respuesta en el prólogo de Avellaneda.
} 
lo apreciable para el autor directamente afectado, lo que, a buen seguro, nos explica la corrección ya efectuada en la segunda edición de Juan de la Cuesta, en la que, como en tantos y tantos pasajes ${ }^{11}$, trataría de subsanarse una falsa errata de impresión.

Prueba de la escasísima difusión del texto de Urrea es cierta observación de Pierre Geneste, quien ha dedicado dos extensos volúmenes al poeta de Épila:

Considérée dans un ensemble aujourd'hui presque entièrement accessible - seule manque à l'appel, parmi les écrits recensés, La Famosa Epila-, l'oeuvre a des caractéristiques $[\ldots]^{12}$.

Pero no terminan aquí los testimonios de interés, pues en el Museo aragonés, otro de los manuscritos preparados por Ustarroz con el propósito de recoger, entre otras cosas, la nómina de las obras escritas por los autores aragoneses, se vuelve a aludir a La famosa Epilia, añadiéndose un importante detalle sobre la adscripción genérica de la obra. Y es que, en el f. $264 \mathrm{v}$, leemos que la Epilia famosa es libro de idéntico género al del Quijote:

La famosa Epilia libro cavalleresco no está impreso ${ }^{13}$.

Recordemos, en este sentido, que la afición de Urrea por los libros de caballerías le había llevado en 1549 a traducir el Orlando furioso de Ariosto, tarea por la que se había ganado, pese a la gran cantidad de reimpresiones, las críticas de buena parte de sus contemporáneos y - cómo nola tajante reprobación de Cervantes ${ }^{14}$, quien, en el propio Quijote, se refiere en tono marcadamente irónico a su torpeza.

Así, en el episodio del escrutinio, leemos la siguiente conversación entre el cura y el barbero, en la que, también de modo indirecto, se burla del capitán Jerónimo de Urrea:

11 Vid. Robert M. Flores, The Compositors of the First and Second Madrid Editions of "Don Quixote" Part I, Londres, Modern Humanities Research Association, 1975.

${ }_{12}$ Cfr. Pierre Geneste, Essai sur la vie et l'oeuvre de Jerónimo de Urrea, Paris, Université de Lille, 1975, 2..$^{\circ}$ vol., pág. 708. De modo significativo, la Epilia famosa no se recoge ni aun en obras como las de Frank Pierce, La poesía épica del Siglo de Oro, Madrid, Gredos, 1968; José Simón Díaz, Bibliografia de la literatura hispánica, Madrid, CSIC, 1982, que se ocupa en el tomo XII, págs. 245-9, de Jerónimo de Urrea; José Manuel Lucía Megías, «Libros de caballerías manuscritos», Voz y Letra, vII, 2, 1996, págs. 61-126; etc.

${ }_{13}$ Nos referimos al ms. 9457 de la BNM, autógrafo de Ustarroz de 1639 que fue originalmente titulado «MUSEO||ANTIGVO I MODERNO||DE LOS HISTORIADORES||DE||ARAGÓN||I||SS CORONA», palabras que se tachan y sustituyen por Museo aragonés. Hay copia de este autógrafo fechada el 26 de agosto de 1734, el ms. 7410 de la BNM.

${ }_{14}$ Vid., por ejemplo, Maxime Chevalier, L'Arioste en Espagne (1530-1650). Recherches sur l'influence $d u$ "Roland furieux", Burdeos, Institut d'Études Ibériques et Ibéro-américaines, 1966, págs. 439-91. 
- [...] y en verdad que estoy por condenarlos no más que a destierro perpetuo, siquiera porque tienen parte de la invención del famoso Mateo Boyardo, de donde también tejió su tela el cristiano poeta Ludovico Ariosto; al cual, si aquí le hallo, y que habla en otra lengua que la suya, no le guardaré respeto alguno; pero si habla en su idioma, le pondré sobre mi cabeza. - Pues yo le tengo en italiano - dijo el barbero-, mas no le entiendo. - Ni aun fuera bien que vos le entendiérades - respondió el cura-, y aquí le perdonáramos al señor capitán que no le hubiera traído a España y hecho castellano; que le quitó mucho de su natural valor $[\ldots]^{15}$.

El pasaje nos da pie a formular, al menos, dos observaciones de interés para el tema que nos ocupa. En primer término, y es obvio decirlo, el hecho de que Cervantes conozca la traducción del Orlando hecha por Urrea refuerza la posibilidad de una alusión a lo que, según leíamos en el manuscrito de Ustarroz, es también «libro cavalleresco», la Epilia famosa del propio Urrea; en segunda instancia, la actitud del novelista ante el maltratado Urrea nos podría facilitar un posible contexto irónico en el que interpretar la alusión a la Hepila del primer capítulo quijotesco; a saber: Amadís añadiría a su nombre el de su patria, al igual que estaría buscándose el engrandecimiento de la patria en la obra caballeresca de Urrea, lo que sitúa a ésta en un plano de importancia evidentemente sobredimensionado, como es la práctica habitual en el discurso irónico de Cervantes.

Esta posibilidad eximiría, en el caso de que entendamos que el antecedente sintáctico al que hace referencia el circunstancial de causa "por Hepila famosa» es el verbo "añadió», del posible anacronismo e incluso falsedad de la influencia ejercida por la Epilia en el Amadís. Importaría sólo engrandecer, con un fin humorístico, lo que tan sólo sería para Cervantes un intranscendente y pretencioso intento de ensalzar la localidad de Épila, tan insignificante para éste en materia de caballerías.

Cabe, no obstante, una segunda lectura, y es que, con idéntico propósito burlesco, el sintagma en cuestión se refiera, mediante una concordancia ad sensum perfectamente recurrente en el Quijote, al verbo «acordándose», del que sería sujeto don Quijote, a quien su locura le lleva a ensalzar el nombre de la Mancha ${ }^{16}$.

$\mathrm{Y}$, con un sentido parcialmente diferenciado de los anteriores, quizá se recoja con la preposición por un valor de locación-causalidad ('a través de'), en el que no se ve necesariamente implicada una idea de 'penetración'.

\footnotetext{
${ }^{15}$ Cfr. ed. cit. (1994), pág. 68. En n. 23 se sugiere que Jerónimo de Urrea «podría ser el "mal traductor" aludido en II, cuando don Quijote visita una imprenta en compañía de don Antonio Moreno (LXII, 1005)».

${ }^{16}$ Cfr. Federico Sánchez Escribano, «Del posible sentido paródico de la Mancha en el Quijote», Anales Cervantinos, VIII, 1959-60.
} 
De esta suerte, el texto, que estaría transmitiendo una lectura del todo apegada al original cervantino, vendría a decir, con el habitual tono de ironía, que la patria de Amadís habría sido, en efecto, 'famosa a través de Épila'; es decir: conocida, en tanto que referente caballeresco, a través de la labor de difusión literaria llevada a cabo por el importante centro cultural de Épila, donde se desarrolló una amplia actividad tanto historiográfica como literaria.

Según nos recuerda Carrasco-Urgoiti:

In the late $1550 \mathrm{~s}$ and $1560 \mathrm{~s}$ the count of Aranda patronized a group of historians and poets, some of whom were related to Jorge de Montemayor. Another interesting influence exerted on this circle was that of the count's kinsman Jerónimo Jiménez de Urrea. A captain of Charles V's army, this prolific writer spent many years away from his native land, living mostly in Italy, but he kept in touch with the Épila circle. Urrea's translations helped considerably to disseminate in Spain new trends in literature $[\ldots]^{17}$.

La presente interpretación descartaría, en consecuencia, la hipótesis de una lectio facilior de Hepilia como topónimo que, presente en la memoria del componedor, le habría llevado a imprimir Hepila en la princeps; y esto porque, sencillamente, el texto de la primera edición de 1605 llevaría a los moldes una lectura idéntica a la del manuscrito autógrafo inicialmente entregado por Cervantes: por Hepila famosa, alusión irónica al poder de difusión cultural del círculo de escritores tan allegado a Urrea, lo que, sin duda, no impediría inferir una alusión indirecta a La Epilia famosa, desde el momento en que, junto a la serie de argumentos expuestos anteriormente, la obra 《related in some manner to the literary group that has been mentioned ${ }^{18}$ ».

Sea de ello lo que fuese, y sin descartar - toda precaución es pocaun posible salto de línea o, incluso, la supresión de una parte del texto manuscrito, lo indiscutible es que, analizado el pasaje desde una u otra opción interpretativa, resulta del todo innecesario rebasar los límites de lo que, por glosar a Blecua, se impone como la única posibilidad factible «en buena ley textual»: la lectura Hepila de la princeps, ya se avale ecdóticamente como lectio facilior (topónimo) de Epilia, ya se acepte la última de las interpretaciones aquí sugeridas. No incurramos, entonces, en esa misma ligereza con la que, cuatrocientos años atrás, "vulgarizaron" la príncipe los copistas encargados de reeditarla en 1605.

\footnotetext{
${ }^{17}$ Cfr. María-Soledad Carrasco-Urgoiti, The moorish novel. "El Abencerraje" and Pérez de Hita, Boston, Twayne, 1976, pág. 62.

18 Ibidem, pág. 62. Vid. al respecto María-Soledad Carrasco-Urgoiti, «Las cortes señoriales del Aragón mudéjar y El Abencerraje», en Homenaje a Casalduero (Madrid, Gredos, 1972), págs. 115-28.
} 